\title{
RADIO OBSERVATIONS OF ANGULAR STRUCTURE
}

\author{
H.P. PALMER \\ (Nuffield Radio Astronomy Laboratories, Jodrell Bank)
}

\section{Introduction}

Complete information about the angular sizes and structures of discrete radio sources would, in principle, require observations in many position angles and at a large number of resolving powers, of which the highest power must resolve the smallest significant features in the sources. Since some sources are known to have components with differing radio spectra, similar amounts of data would be required at each of several wavelengths. One would then be able to examine statistically the relation between fringe visibility and resolving power at two or three wavelengths, and hence search for structural differences between different types of discrete sources.

\section{The Data}

The information on the size and structure of radio sources available at present is very incomplete by these ideal standards. What is known could be summarised in many ways. It is considered here in three ranges of angular size (A) $1^{\prime} \rightarrow 1^{\prime \prime}$, (B) $1^{\prime \prime} \rightarrow 0 \cdot 1^{\prime \prime}$, and $(\mathrm{C})<0 \cdot 1^{\prime \prime}$.

\section{A. MEASUREMENTS USING RESOLVING POWERS BETWEEN $1^{\prime}$ AND $1^{\prime \prime}$ OF ARC}

Almost all the measurements in this range have been made with interferometers using baselines between 2000 and 61100 wavelengths long, at frequencies from 38-2695 $\mathrm{MHz}$. The extent of these measurements has been summarised in columns 3 and 4 of Table 1. For a small number of sources data are available showing resolution over a fair range of baselines and at a number of position angles. In some cases measurements were made of the phases as well as of the amplitudes of the fringe patterns. Fourier inversions were then performed and detailed structures of the sources determined uniquely. Approximately 80 such maps have been published so far of such detailed structures. In a similar number of cases there are not sufficient data for this process, but it is possible to fit to the data plausible source models; normally these have double structures. The results of such analyses are described as 'maps' or 'models' in Table 1, column 3. It will be seen that most of these data come from the observations at Owens Valley $(\mathbf{1}, \mathbf{2})$ and a certain amount has already been published 


\section{Table 1}

Interferometric measurements with resolving powers between $1^{\prime}$ and $1^{\prime \prime}$ of arc

(1)

Wavelength

(ג)

$\begin{array}{cl}7.8 \mathrm{~m} & \geqslant 2^{\prime \prime} \\ 1.9 \mathrm{~m} & 47^{\prime \prime}-1^{\prime \prime} \\ 30 \mathrm{~cm} & >40^{\prime \prime} \\ 21 \mathrm{~cm} & >20^{\prime \prime} \\ 21+70 \mathrm{~cm} & >10^{\prime \prime} \\ 11 \mathrm{~cm} & >3^{\prime \prime}\end{array}$

(3)

No. of Structures Reported

$\begin{aligned} & - \\ & 6 \text { models } \\ & 62 \text { models } \\ \sim & 60 \text { maps } \\ \simeq & 10 \text { maps } \\ \simeq & 10 \text { models } \\ + & \text { one map }\end{aligned}$

$\begin{array}{cl}18 & \text { Jodrell Bank-Malvern (12) } \\ 378 & \text { Jodrell Bank (7) } \\ 133 & \text { Owen's Valley (1) } \\ 540 & \text { Owen's Valley (2) } \\ - & \text { Cambridge }(\mathbf{3 , 4}, \mathbf{5}) \\ 146 & \text { Greenbank (6) }\end{array}$

(4)

No. of Size Estimates

(5)

Observatories

Resolvin

Powers

from the 1-mile telescope at Cambridge $(3,4,5)$. The resolving powers of these instruments are less than 10000 wavelengths, so that angular sizes bigger than about $5^{\prime \prime}$ are detected. Much smaller numbers of sources have been studied at higher resolving powers, when some variation of the baseline is often obtained by the fore-shortening that occurs as the sources move across the sky. Data have been published for 10 sources observed at $11 \mathrm{~cm}$ at Greenbank (6) and for 6 observed at $2 \mathrm{~m}$ at Jodrell Bank (7). More than $60 \%$ of these sources are double.

The second class of data from these observations comprises approximate estimates of the overall angular sizes of sources which have been inferred from limited observations at one or a few baselines. Such estimates are available for much larger numbers of sources as shown in column 4.

A second technique which has been used to give diameter information in this range and somewhat below is that of lunar occultation. After the initial striking success of this method with the first quasar $3 \mathrm{C} 273$ later occultations of that quasar have been studied extensively. This series of occultations is now complete, and this quasar will not be occulted again for 12 years. Results from occultations of fewer than a dozen other sources have so far been published, although Hazard (8) has recently reported 5 examples of a very large number of occultations observed at frequencies from 41-610 $\mathrm{MHz}$ with the 1000-ft. telescope at Arecibo.

\section{B. MEASUREMENTS USING RESOLVING POWERS BETWEEN$$
1^{\prime \prime} \text { AND } 0 \cdot 1^{\prime \prime} \text { OF ARC }
$$

Two different techniques of observing sources have contributed to the information in the range from 1" to $0 \cdot 1^{\prime \prime}$ of arc. Interferometric measurements from Jodrell Bank (9) and from the Jodrell Bank-Malvern interferometer $(10,11,12)$ for about 40 sources are summarised in Table 2 .

The second method of obtaining diameter information in this size range uses the scintillation of sources due to the motion of irregularities in the interplanetary solar 
Table 2

Measurements with resolving powers between $1^{\prime \prime}$ and $0 \cdot 1^{\prime \prime}$ of arc

\begin{tabular}{lcccl}
\multicolumn{1}{c}{ Technique } & $\begin{array}{c}\text { Wavelength } \\
(\lambda)\end{array}$ & $\begin{array}{c}\text { No. of Structures } \\
\text { reported }\end{array}$ & $\begin{array}{c}\text { No. of Size } \\
\text { estimates }\end{array}$ & Observatories \\
$\begin{array}{l}\text { Interplanetary } \\
\text { scintillation }\end{array}$ & $1.7 \mathrm{~m}$ & - & $\begin{array}{c}45 \\
(140 \text { selected } \\
\text { sources observed) }\end{array}$ & Cambridge (14) \\
$\begin{array}{l}\text { Interplanetary } \\
\text { scintillation }\end{array}$ & $70 \mathrm{~cm}$ & - & $\begin{array}{c}70 \\
(138 \text { selected } \\
\text { sources observed) }\end{array}$ & Arecibo (15) \\
$\begin{array}{l}\text { Interplanetary } \\
\text { scintillation }\end{array}$ & $70 \mathrm{~cm}$ & - & $\begin{array}{c}10 \\
\text { (Random sample of }\end{array}$ & Arecibo (15) \\
$\begin{array}{l}\text { Radio link } \\
\text { Interferometer }\end{array}$ & $70 \mathrm{~cm}$ & 4 models & $\begin{array}{c}27 \text { sources observed) } \\
12\end{array}$ & Jodrell Bank (9) \\
& $21 \mathrm{~cm}$ & 4 models & 38 & J.B.-Malvern (10, 11, 12)
\end{tabular}

wind. This method was discovered by Hewish et al. (13) and reported to the last IAU. It has since been exploited by the Cambridge group, and by Cohen and his colleagues at Cornell/Arecibo. A measure of the angular diameter of a source is obtained from the ratio of the scale of the diffraction pattern at the Earth to its distance from the diffracting screen. Under simple circumstances this is the ratio of the scale of irregularities in the plasma of the solar wind to one AU, i.e. about $0 \cdot 3^{\prime \prime}$ of arc. Radio sources smaller than this angular size scintillate while larger ones do not. On this basis Little (14) has made a survey at $178 \mathrm{MHz}$ of the scintillation of $1403 \mathrm{CR}$ sources which are of adequate flux density and which pass sufficiently close to the Sun. About $30 \%$ have components smaller than $0 \cdot 3^{\prime \prime}$ of arc. Cohen et al. (15) have used the Arecibo telescope to observe 140 sources at one or more of the frequencies 195, 430 and $611 \mathrm{MHz}$. These sources were not confined to the 3CR catalogue, but were selected as likely to show scintillation, which was detected for 70 of them. They also surveyed a smaller but unselected sample of 27 sources at $430 \mathrm{MHz}$, and found that 10 of these scintillated. They concluded that approximately $40 \%$ of sources whose flux at $408 \mathrm{MHz}$ exceeds $2 \mathrm{f}$.u. emit a detectable fraction of their flux from a region smaller than $0 \cdot 3^{\prime \prime}$ diameter, and that they could determine the percentage of the total flux which was emitted by these small components.

A greater resolution becomes possible when observations are made closer to the Sun, and at lower frequencies. The diffracting screen becomes phase thick, the scale decreases, and the critical angle decreases. Under these circumstances Cohen et al. (16) have used the frequency spectrum of the scintillations to determine the angular scale. They show, e.g., that the angular diameter of $3 \mathrm{C} 138$ is about $0 \cdot 15^{\prime \prime}$, in agreement with Little and Hewish (17), while the diameter of 3C 273B is less than 0.02 " (15). Complications arise when the phase screen becomes thick and when its Fresnel distance becomes comparable with an astronomical unit. There has been some 
controversy about the interpretation of measurements made under these conditions, and the analyses of Little and Hewish $(\mathbf{1 7}, \mathbf{1 8})$ and Salpeter $(\mathbf{1 9})$ should be consulted.

Some information about the structure of sources can also be deduced if one knows the properties of the solar wind well enough, and finds variations in the power spectra of the scintillations which correlate with changes in the direction and angular distance between the sources and the Sun. For instance, Rickett has just shown that at 408 $\mathrm{MHz}$ the quasar 3C446 (red-shift $z=1.402$ ) is probably elongated, with a size of about $0 \cdot 4^{\prime \prime}$ in position angle $94^{\circ}$, by about $0 \cdot 2^{\prime \prime}$ in p.a. $34^{\circ}$. This agrees with the detailed analysis of the $21-\mathrm{cm}$ interferometric measurements on this source made between Jodrell Bank and Malvern $(\mathbf{2 0 , 2 3 )}$.

\section{MEASUREMENTS OF RADiO SOURCES SMALLER THAN $0 \cdot 1^{\prime \prime}$ OF ARC}

In 1963 Slish (21) and Williams (22) predicted from spectral considerations that some quasars would be found to have diameters of order 0.01 " of arc, while the discovery of secular changes of radio flux implied that some sources were smaller still. The Jodrell Bank to Malvern 21-cm observations were therefore extended to 11 and $6 \mathrm{cms}(12)$, where 4 sources were still unresolved with a baseline of $2 \times 10^{6} \lambda$ (Table 3 ).

\section{Table 3}

\section{Interferometric measurements of radio sources smaller than $0 \cdot 1^{\prime \prime}$ of arc}

\begin{tabular}{|c|c|c|c|c|}
\hline Wavelength & $\begin{array}{l}\text { Baseline } \\
\text { Length }\end{array}$ & Resolving power & $\begin{array}{c}\text { No. of size } \\
\text { estimates }\end{array}$ & Observatories \\
\hline $65 \mathrm{~cm}$ & $183 \mathrm{~km}$ & $0.3^{\prime \prime}\left(2.7 \times 10^{5} \lambda\right)$ & 12 & Algonquin-Dominion R.A.O. \\
\hline $65 \mathrm{~cm}$ & $3074 \mathrm{~km}$ & $0.02^{\prime \prime}\left(4.6 \times 10^{6} \lambda\right)$ & 8 & Algonquin-Penticton (23) \\
\hline $50 \mathrm{~cm}$ & $250 \mathrm{~km}$ & $0.2^{\prime \prime}\left(4.6 \times 10^{5} \lambda\right)$ & 4 & Greenbank-Maryland Point \\
\hline $50 \mathrm{~cm}$ & $2000 \mathrm{~km}$ & $0.02^{\prime \prime}\left(4.0 \times 10^{6} \lambda\right)$ & 1 & Greenbank-Arecibo ${ }^{a}$ \\
\hline $18 \mathrm{~cm}$ & $840 \mathrm{~km}$ & $0.02^{\prime \prime}\left(4.7 \times 10^{6} \lambda\right)$ & 13 & Greenbank-Haystack ${ }^{a}$ \\
\hline $18 \mathrm{~cm}$ & $4000 \mathrm{~km}$ & $0.003^{\prime \prime}\left(1.95 \times 10^{7} \lambda\right)$ & $\begin{array}{c}1 \\
(3 \mathrm{C} 273 \mathrm{~B})\end{array}$ & Greenbank-Berkeleya \\
\hline $13 \mathrm{~cm}$ & $1200 \mathrm{~km}$ & $0.01^{\prime \prime}\left(9 \times 10^{6} \lambda\right)$ & $\begin{array}{c}1 \\
(3 \mathrm{C} 273 \mathrm{~B})\end{array}$ & Australia (28) \\
\hline $11 \mathrm{~cm}$ & $127 \mathrm{~km}$ & $0.05^{\prime \prime}\left(1.1 \times 10^{6} \lambda\right)$ & 35 ( 4 models) & Jodrell Bank-Malvern (12) \\
\hline $6 \mathrm{~cm}$ & $127 \mathrm{~km}$ & $0.025^{\prime \prime}\left(2.1 \times 10^{6} \lambda\right)$ & 4 & Jodrell Bank-Malvern (12) \\
\hline
\end{tabular}

a Private communications at first or second hand.

This equipment used three broadband radio links, and it seemed that for baselines longer than 100 miles an interferometer using rubidium frequency standards and video tape recorders would provide a more satisfactory and economic method of extending the observations. Several systems have been constructed in different parts of the world, and the results known to the author are summarised in Table 3. The first correlation was observed at a wavelength of $65 \mathrm{~cm}$ by the Canadians (23) using a 
baseline 4.6 million wavelengths long. Similar observations were made between Greenbank and several other observatories. It has also been reported that during the observations of $\mathrm{OH}$ emissions $(\lambda=18 \mathrm{~cm})$ made at 19.5 million wavelengths between Greenbank and Berkeley, correlation was measured for 3C 273B. This gives a size of less than 0.003" of arc for this quasar. 3C 273 has also been observed in Australia at 9 million wavelengths with a post-detector correlator system (28).

The data from tape-recording interferometers known at the time of writing refer to $3 \mathrm{C} 273 \mathrm{~B}$ and 15 other sources. Seven of these are unresolved at one or more of the frequencies of observation. The remaining sources appear to have a fringe visibility significantly less than unity but there are some differences between the results, which may represent actual differences of source size with frequency. The observations reported from tape-recording interferometers have not yet given any indication of the structure of these very small sources.

\section{The Distribution of Diameters}

The first major diameter surveys at Cal. Tech. at $960 \mathrm{MHz}(\mathbf{1})$ and at Jodrell Bank at $159 \mathrm{MHz}(24)$ were made as complete as possible in order to reduce selection effects. Fifty-five of the sources observed in both surveys had galactic latitudes $>12^{\circ}$,

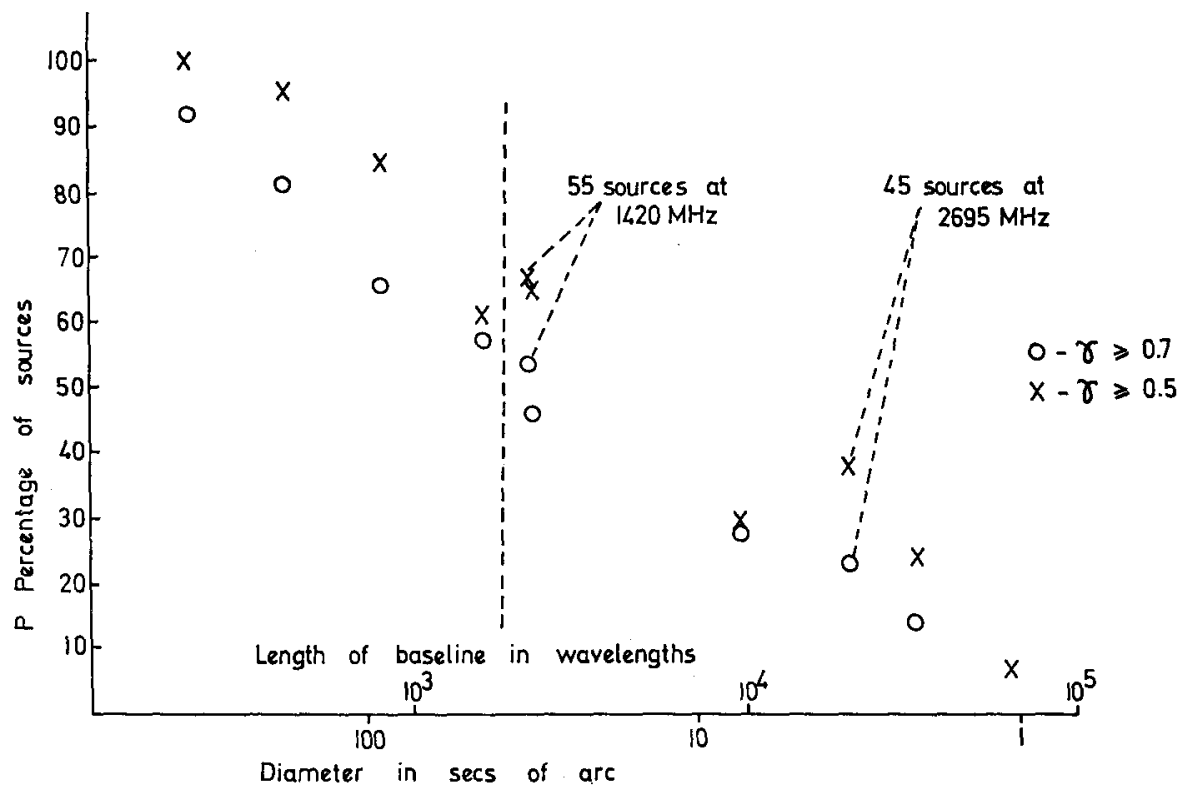

FIG. 1. Data from Maltby and Moffet (1) and Allen et al. (24) have been used to find the percentage $P$ of sources with fringe visibility $(a) \gamma \geqslant 0.7$ and $(b) \gamma \geqslant 0.5$. These observations refer to the restricted group (described in the text) of 55 sources. The points derived from Moffet's data lie to the left of the dashed line. 
and flux densities at $178 \mathrm{MHz}>12 \mathrm{f}$.u. Figure 1 shows the percentage of sources In this sample having fringe visibilities $\gamma \geq 0.7$ and $\gamma \geq 0.5$ at several baselines (25). it will be seen that more than half of the sources were partially resolved by baselines of 10000 wavelengths or more, and that there is good agreement between the two surveys, in spite of the frequency difference of a factor of 6 . The diameter surveys of Fomalont at $1420 \mathrm{MHz}(2)$ and of Clark and Hogg at $2695 \mathrm{MHz}$ (6) included most of these 55 sources. Figure 2 shows that points derived from their new data agree well

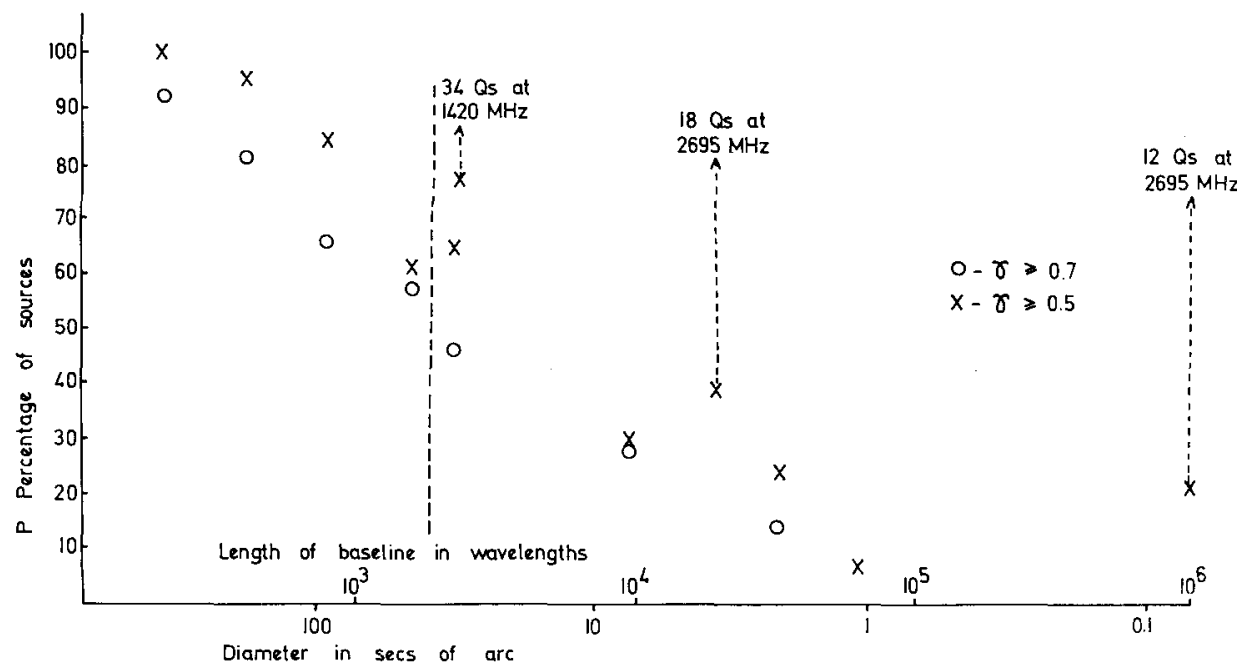

FIG. 2. This diagram includes values of $P$ (as described for Figure 1) which have been derived from the data of Fomalont $(1420 \mathrm{MHz} ; 2)$ and Clark and Hogg $(2695 \mathrm{MHz} ; 6)$.

with the earlier results, although the frequency range is now increased by a further factor of 3. So it appears that the angular sizes of most radio sources do not change markedly with frequency, at least as far as this range of resolution is concerned.

The data at higher resolving powers are much less complete, for when a new instrument is first set up it is used initially to observe those sources most likely to show small scale structure. No complete surveys unaffected by selection effects have yet been made of sources with sizes smaller than 1" of arc. The data in Tables 2 and 3 are therefore strongly biased towards sources which have very small diameters, and cannot be used to extend Figure 2 beyond resolving powers of 60000 wavelengths. It is interesting to note, however, that of the 39 sources observed at $11 \mathrm{~cm}$ in the Jodrell Bank-Malvern programme, $\sim 20$ were unresolved. A further 6 contained an unresolved component radiating more than $50 \%$ of the flux. In several cases it is now clear that these small diameter components have much flatter radio spectra than the remainder of the source, so that at shorter wavelengths they radiate a larger fraction of the total emission (11). 
It is difficult to include in Figure 2 diameter data derived from scintillation measurements, partly because the relationship between scintillation index and fringe visibility is not known in detail, and partly because of selection effects. The two sets of data available for unselected samples of sources (140 sources studied by Hewish and Little and 27 sources observed by Cohen $e t$ al.) both show that approximately $30 \%$ of discrete sources contain components smaller than $0 \cdot 3^{\prime \prime}$ of arc. These components may, however, be contributing only small fractions of the total emission from the sources. The scintillation data should therefore be compared with values of $P$ calculated from interferometric measurements for ranges of fringe visibility going to lower limits of $\gamma$ such as $\gamma>0.1$ or $\gamma>0.3$. The results of the two types of measurements are probably not in contradiction, for we can evidently expect many of the sources which are resolved according to Figures 1 and 2 to contain some structure of much smaller angular size. It is clear that more complete diameter surveys are needed at the higher resolving powers, particularly at wavelengths of $1 \mathrm{~m}$ and longer. Scintillation and interferometric data must be presented in the same way before they can usefully be compared, although the present data may already allow an extension of the preliminary examination by Moffet and Palmer (26) of the radio spectra of the small and large components known to exist in most radio sources.

\section{The Distribution of Diameters for Quasars}

Schmidt (27) has discussed the optical and radio-luminosity functions of 40 quasars. Thirty-four of these quasars were observed by Fomalont (2), 18 by Clark and Hogg (6), and 12 by Palmer et al. (12). Although the complete sample of quasars was not observed, values of $P$ have been calculated on the assumption that the sources which were not observed would have been given values of $\gamma<0 \cdot 5$. These values are shown in Figure 3, on which the dashed lines show how $P$ would have changed if all the missed sources had given fringe visibilities $\gamma>0 \cdot 5$. The paucity of the data makes these values unreliable, but they suggest that at short wavelengths the radio emission of quasars usually comes from regions whose linear sizes are 10 to 1000 times smaller than the emitting regions associated with the majority of radio sources. The smallest sources also tend to show time variations in their radio flux, and changes in the brightness and other properties of the optical object.

\section{Conclusion}

The diameter measurements reviewed in this article suggest, in summary, that some quasars, usually those which are optically active, emit radio energy from a very small, isolated region which may be described as a 'core'. Almost all the remaining quasars give radio emission from two separated regions, and there is often a 'core' in one or both of these regions. In several cases the radio emission from these cores is known 


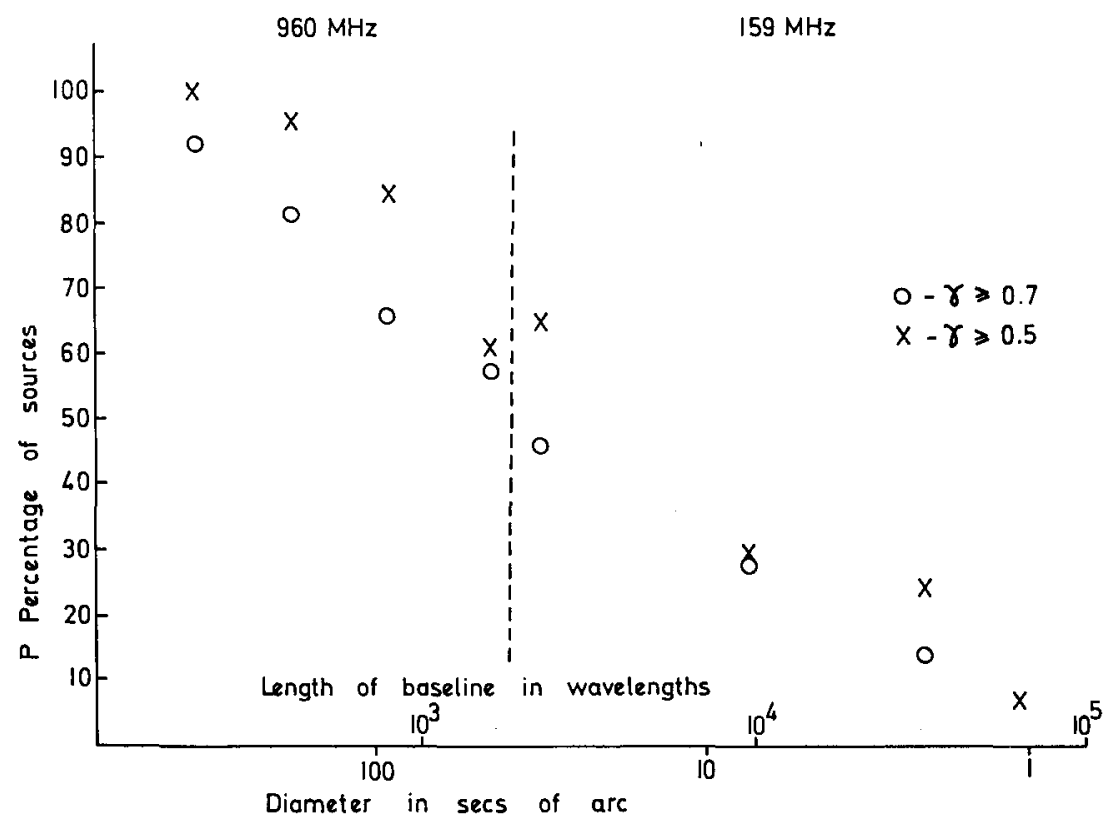

Fig. 3. Values of $P$ (as defined for Figure 1) derived for those quasars in the list of 40 discussed by Schmidt (27) for which diameter data are available.

to vary with time, particularly at short wavelengths. The radio spectra of these cores are relatively flat, or the energy emitted by the core may even increase at short wavelengths. Many radio galaxies are also known to have relatively weak radio cores at wavelengths longer than $1 \mathrm{~m}$, but the measurements of radio spectra suggest that, except in special cases such as the Seyfert galaxy NGC 1275, these cores do not usually predominate at shorter wavelengths.

\section{Acknowledgments}

I thank many colleagues who have helped with the preparation of this review, by discussion and by advance information about recent work. I also acknowledge gratefully the assistance of Professor F. Graham Smith, who read the paper in Prague, and of Mr. W. Donaldson, who discussed many points at length, and also prepared the tables and figures.

\section{References}

1. Maltby, P., Moffet, A.T. (1962) Astrophys. J., Suppl. 7, 141.

2. Fomalont, E. B. (1967) Astrophys. J., Suppl. No. 138.

3. Ryle, M., Elsmore, B., Neville, A.C. (1965) Nature, 205, 1259.

4. Ryle, M., Elsmore, B., Neville, A.C. (1965) Nature, 207, 1024.

5. Kenderdine, S., Ryle, M., Pooley, G.G. (1966) Mon. Not. Roy. astr. Soc., 134, 189. 
6. Clark, B. G., Hogg, D.E. (1966) Astrophys. J., 145, 21.

7. Rowson, B. (1963) Mon. Not. Roy. astr. Soc., 125, 177.

8. Hazard, C., Gulkis, S., Bray, A.D. (1967) Astrophys. J., 148, 669.

9. Anderson, B., Donaldson, W. (1967) Mon. Not. Roy. astr. Soc., 137, 81.

10. Adgie, R.L., Gent, H., Slee, O.B., Frost, A.D., Palmer, H.P., Rowson, B. (1965) Nature, 208, 275.

11. Barber, D., Donaldson, W., Miley, G.K., Smith, H. (1966) Nature, 209, 753.

12. Palmer, H.P., Rowson, B., Anderson, B., Donaldson, W., Miley, G. K., Gent, H., Adgie, R.L., Slee, O. B., Crowther, J.H. (1967) Nature, 213, 789.

13. Hewish, A., Scott, P.F., Wills, D. (1964) Nature, 203, 1214.

14. Little, L.T. (1967) Ph.D. Thesis, Cambridge University.

15. Cohen, M. H., Gundermann, E.J., Harris, D. E. (1967) Astrophys. J., 150, 767.

16. Cohen, M.H., Gundermann, E.J., Hardebeck, H.E., Sharp, L.E. (1967) Astrophys. J., 147, 449.

17. Little, L.T., Hewish, A. (1966) Mon. Not. Roy. astr. Soc., 134, 221.

18. Little, L. T., Hewish, A. in press.

19. Salpeter, E. E. (1967) Astrophys. J., 147, 433.

20. Gent, H., Miley, G.K., Rickett, B. in preparation.

21. Slish, V.I. (1963) Nature, 199, 682.

22. Williams, P.J.S. (1963) Nature, 200, 56.

23. Broten, N.W., Legg, T.H., Locke, J.L., McLeish, C.W., Richards, R.S., Chisholm, R.M., Gush, H.P., Yen, J. L., Galt, J.A. (1967) Nature, 215, 38.

24. Allen, L.R., Anderson, B., Conway, R.G., Palmer, H.P., Reddish, V.C., Rowson, B. (1962) Mon. Not. Roy. astr. Soc., 124, 477.

25. Allen, L. R., Hanbury Brown, R., Palmer, H.P. (1962) Mon. Not. Roy. astr. Soc., 125, 57.

26. Moffet, A.T., Palmer, H.P. (1965) Observatory, 85, 45.

27. Schmidt, M. Preprint.

28. Gubbay, J.S., Robertson, D.S. (1967) Nature, 215, 1157.

\section{DISCUSSION}

Hughes: A large percentage of extragalactic radio sources are known to consist of more than one emission region, and it has been suggested that less than about $20 \%$ can be described as either simple or 'core and halo' sources. However, most of the observations that lead to this interpretation have been made with interferometers at a few fixed frequencies only, and some caution may be necessary before making a generalization for all frequencies. This may be illustrated by reference to the spectrum of the source 4C 50.11/LHE 100/NRAO 150. The spectrum is similar to those of 3C 279 (a quasistellar source) and 3C 84 (the Seyfert Galaxy NGC 1275) but has a double hump at the higher frequencies. Three components can be recognized:

(1) Component $A$ with a spectral index of -0.5 between 78 and $100 \mathrm{MHz}$.

(2) Component $B$ which has a rising spectrum with an index of about +2.5 , a peak at about $300 \mathrm{MHz}$ and, above that, a falling spectrum with an index of -0.5 .

(3) Component $\mathrm{C}$ which is similar to $\mathrm{B}$ but with a peak at about $6000 \mathrm{MHz}$.

Component $\mathrm{C}$ shows no appreciable broadening of the $3^{\prime}$ of arc beam of the $150 \mathrm{ft}$. telescope at the Algonquin Radio Observatory and the mean position is separated from that of component B, as determined at the U.S. National Radio Astronomy Observatory, by $46^{\prime \prime}$ of arc, with combined errors of about $33^{\prime \prime}$ of arc. The lower frequency $4 \mathrm{C}$ position of component $\mathrm{A}$ is near to the suggested centre of components $\mathrm{B}$ and $\mathrm{C}$, though the error bars overlap the two positions. Hence it appears that the object is at least a double, and quite possibly a triple source. But if measurements were made at frequencies near to the peaks at 300 or $6000 \mathrm{MHz}$, or below about $100 \mathrm{MHz}$ then the source would only appear as a simple single source; close to the minima in the spectrum at about 100 and $3500 \mathrm{MHz}$, the source would appear to have two equal components, whilst for small frequency displacements about these values the source would be described as an unequal double. It is quite obvious that before generalizations are made regarding the complexity of a source, interferometer 
observations are required at a number of frequencies, and the spectrum examined in detail over a very large frequency range.

Locke: A long base-line interferometer has been operating in Canada at a frequency of $488 \mathrm{MHz}$. Antennas at Penticton and Algonquin Park are used with independent local oscillators. At the spacing of $3074 \mathrm{~km}$ only 3 out of the 30 sources observed remain unresolved. They are PKS 1127-14, 3 C 345 and CTA 102 which therefore have angular diameters $<0.01$ " of arc. CTA 21 and 3C 273B are partially resolved with $\gamma=0.5$ and $3 C 279,286,309.1$ have angular diameters of 0.03 " of arc.

Kellermann: Another long base-line interferometer has been working between Hat Creek, Calif. and N.R.A.O., Green Bank. At a wavelength of $18 \mathrm{~cm}$ some sources are unresolved which have been resolved at the longer wavelength in Canada. It is clear that the structure changes with wavelength. 